\title{
Theaterarbeit und kulturelle Bildung
}

\author{
Steffi Robak
}

Online publiziert: 27. Juni 2015

(c) Die Autor(en) 2015. Dieser Artikel ist auf Springerlink.com mit Open Access verfügbar.

\section{Rezension zu:}

Ömer Adigüzel, Ute Handwerg, Gerd Koch (Hg.) (2014). Theater und community - kreativ gestalten. Deutsch-Türkische Kooperationen in der Kulturellen Bildung. Kopaed, München, 474 Seiten, 24,80 €, ISBN 978-3-86736-342-6

Die Aspekte Interkulturalität und Migration sind in der Theaterarbeit, so der Ausgangspunkt des vorliegenden Bandes, und der damit verbundenen kulturellen Bildung, lange ignoriert worden. Der Band dokumentiert aktuelle Ansätze, Entwicklungen, einige theoretische Reflexionen, vor allem aber die Entwicklung der Theaterpädagogik und des kreativen Dramas. Es werden insbesondere Potenziale für den schulischen und außerschulischen Kontext geschildert, über Theaterarbeit Begegnung und Verstehen der Schülerinnen und Schüler sowie der Jugendlichen proaktiv zu gestalten. Seit 2010/2011 werden die Begriffe interkulturell, transkulturell, transnational, hybridkulturell, postmigrantisch dafür ausgelotet (S. 378). Besonders hybridkulturelle und postmigrantische Konzeptionen bergen demnach Möglichkeiten, Durchlässigkeiten, Vermischungen und in der Konsequenz Neuformungen von Kulturen zu thematisieren. Bildungsgehalte des interkulturellen Theaters werden besonders in der Ausgestaltung der Leitbegriffe Differenz, Alterität und Diversität gesehen (S. 381).

Insgesamt finden sich leider nur angedeutete erwachsenenpädagogische Gestaltungsansätze. Die Beiträge sind aber durchaus anregend, um über Fragestellungen für kulturelle Weiterbildung nachzudenken.

Unter der Perspektive Theater und Community stellen verschiedene Autorinnen und Autoren aus Praxis und Wissenschaft Beispiele einer partizipativen künstlerischen Praxis mit Bildungsgehalten vor. Diese sind das Ergebnis einer jahrelangen

Prof. Dr. S. Robak $(\square)$

Leibniz Universität Hannover,

Schloßwender Str. 1,

30159 Hannover, Deutschland

E-Mail: steffi.robak@ifbe.uni-hannover.de 
engen Zusammenarbeit zwischen den deutschen und türkischen Arbeitsgemeinschaften für Spiel und Theater. Spiel, Drama und Theaterpädagogik werden in ihren Ressourcen für Verständigung, Partizipation und Gemeinschaft im Kontext des internationalen Austausches dargestellt.

In den überwiegend kurzen Beiträgen werden Entwicklungen der Theaterpädagogik in Deutschland und des kreativen Dramas in der Türkei beschrieben. Diese sind zwei- und in Teilen dreisprachig, auf Deutsch, Türkisch und Englisch verfasst, sodass eine breite Rezeption möglich ist. Sie sind sowohl für die konkrete kulturelle Bildungsarbeit ein Gewinn, als auch für weitere Überlegungen für den Fortbildungsbereich. Der Band gliedert sich in vier Teile: geschichtliche Anfänge und berufliche Erinnerungen, gegenwärtige Drama- und Theaterarbeitsfelder, exemplarische Beispiele communitybezogener kultureller Bildung, kultur- und bildungsbezogene Konzeptionen und Perspektiven, internationale Übereinkunft über professionelles Verhalten und Ethik. Es ist angekündigt, dass die Perspektive über die gesamte Lebensspanne reicht, faktisch wird der schulische und Jugendbildungsbereich adressiert. Die Einleitungen, als persönliche Geschichten formuliert, erfolgen essayistisch oder als Interview, sodass einerseits der Zugang zum Buch niedrigschwellig ist; Praktiker bekommen so einen schnellen Zugang. Andererseits wird erlebbar, welchen Stellenwert Theaterpädagogik im Leben der Kulturschaffenden und -vermittelnden hat und wie eng individuelle Bindungen an mehrere Kulturen sein können, die die künstlerische und bildnerische Arbeit inspirieren. Die z. B. von Marlies Krause vorgestellten Projekte, die in der Türkei angesiedelt sind und von den Hochschulen ausgingen (S. 88), sind sehr interessant, innovativ und meist auf konkrete öffentliche Räume bezogen. Auch für Angebote der Erwachsenenbildung bieten sich einige Anregungen. Es findet sich ein Beispiel, das gelungene Kooperationen mit Volkshochschulen abbildet (S. 97 f.). Auch die weiteren Beispiele aus dem Jugendbildungsbereich (Beitrag von Klaus Hoffmann), die Entwicklungen von darstellendem Spiel inner- und außerhalb von Schule in der Türkei (Zera Ipsiroglu), die auch an deutschen Universitäten erprobt wurden, zeigen Möglichkeiten, wie erfahrene Gewalt, Diskriminierung, Aggressivität bewältigt und andere Fähigkeiten des sozialen Umgangs und der Beziehungsgestaltung geübt werden können. Praktizierte Inszenierungen verlangen Rollenwechsel, Perspektivübernahme, bis hin zum Reflektieren von Geschlechterrollen in den unterschiedlichen Kulturen (S. 134). Besonders die diskursiven Möglichkeiten der Auseinandersetzung über konkrete gesellschaftliche Konfliktlagen scheinen ausbaufähig. Die Optionen grenzüberschreitenden Handelns durch Theater/Drama sind sowohl für Kinder und Jugendliche als auch Erwachsene nicht ausgeschöpft (S. 170). Möglichkeiten der internationalen Verständigung über ästhetische Wahrnehmung und Gestaltung und die Thematisierung politischer und sozialer Verhältnisse sind noch nicht ausgelotet (Beitrag von Jutta Heppekausen).

Während die vielfältigen Beispiele der gemeinsamen interkulturellen Theaterarbeit eine interessante Ausdifferenzierung für den Jugendbildungsbereich zeigen, bleiben die Übergänge für den Bereich der Erwachsenenbildung bis zuletzt nur angedeutet, so z. B. in dem Beitrag, der beschreibt, wie ein Dorf Theater spielt. Eine Ausarbeitung und theoretische Aufarbeitung inter- und transkultureller Bildungsarbeit für darstellende Formen wäre auch für den Erwachsenenbildungsbereich wünschenswert und notwendig. 
Open Access Dieser Artikel wird unter der Creative Commons Namensnennung 4.0 International Lizenz (http://creativecommons.org/licenses/by/4.0/deed.de) veröffentlicht, welche die uneingeschränkte Nutzung, Verbreitung und Wiedergabe für beliebige Zwecke erlaubt, sofern Sie den/die ursprünglichen Autor(en) und die Quelle ordnungsgemäß nennen, einen Link zur Creative Commons Lizenz beifügen und angeben, ob Änderungen vorgenommen wurden. 